\title{
Infiltração de sal de alumínio em fibras de sisal para obtenção de fibras de alumina
}

\section{(Infiltration of aluminum salt into sisal to produce alumina fibers)}

\author{
T. E. Andrade Jr. ${ }^{1}$, A. E. Martinelli ${ }^{1}$, D. M. A. Melo ${ }^{I}$, R. M. Nascimento ${ }^{I}$, \\ C. Rambo ${ }^{2}$, H. Sieber ${ }^{2}$, P. Greil ${ }^{2}$ \\ ${ }^{1}$ Departamento de Química, Laboratório de Química Inorgânica e Materiais \\ Universidade Federal do Rio Grande do Norte, Campus Lagoa Nova s/n \\ Natal, RN 59072-970 \\ ${ }^{2}$ Department of Materials Science (III) - Glass and Ceramics \\ University of Erlangen-Nuernberg \\ Erlangen, Germany D-91058
}

\begin{abstract}
Resumo
O sisal é um recurso estratégico para a região Nordeste e, particularmente, para o estado do Rio Grande do Norte, por ser uma cultura renovável e adaptada às condições do semi-árido. Em virtude das condições adversas de clima e solo, o sisal é, em algumas regiões, o único produto agrícola rentável passível de plantio. Agregar valor aos produtos manufaturados a partir do sisal contribui não só para o desenvolvimento científico e tecnológico da região, como também para a geração de renda para a população dos municípios potiguares produtores de sisal. Da planta extraem-se fibras ligninocelulósicas utilizadas na produção artesanal de cordas e industrial de mantas e tapetes. Uma outra alternativa é o aproveitamento da estrutura da fibra para a produção de fibras de alumina $\left(\mathrm{Al}_{2} \mathrm{O}_{3}\right)$ pela biomodelagem. Este processo consiste na reprodução da estruturas natural do material de partida. O objetivo deste trabalho foi estudar as condições de infiltração da fibra de sisal com sal de alumínio para posterior conversão da estrutura em fibras de alumina. Inicialmente, as fibras de sisal foram pré-tratadas com hidróxido de sódio, infiltradas com uma solução saturada de sal de alumínio $\left(\mathrm{Al}_{2} \mathrm{Cl}_{6}\right)$ e sinterizadas entre $1400{ }^{\circ} \mathrm{C}$ e $1650{ }^{\circ} \mathrm{C}$. A caracterização das fibras de alumina foi feita por difração de raios X e microscopia eletrônica de varredura. Os resultados mostraram a conversão completa apenas da superfície da fibra de sisal em $\alpha-\mathrm{Al}_{2} \mathrm{O}_{3}$, resultando em baixa resistência ao manuseio. Novos métodos de infiltração são propostos com o objetivo de reproduzir-se a estrutura interna da fibra de sisal, resultando em fibras materiais com melhor resistência mecânica.
\end{abstract}

Palavras-chave: fibras de alumina, sisal, biomodelagem.

\begin{abstract}
Sisal is a renewable agricultural resource adapted to the hostile climatic and soil conditions particularly encountered in the semi-arid areas of the state of Rio Grande do Norte, located in Northeastern Brazil. Consequently, sisal has played a strategic role in the economy of the region, as one of few options of income available in the semi-arid. Find new options and adding value to products manufactured from sisal are goals that contribute not only to the scientific and technological development of the Northeastern region, but also to the increase of the family income for people that live in the semi-arid areas where sisal is grown. Lignocellulosic fibers are extracted from sisal and commonly used to produce both handcrafted and industrial goods including ropes, mats and carpets. Alternatively, added-value products can be made using sisal to produce alumina fibers $\left(\mathrm{Al}_{2} \mathrm{O}_{3}\right)$ by biotemplating, which consists in the reproduction of the natural fiber-like structure of the starting material. The objective of the study reported herein was to evaluate the conditions necessary to infiltrate aluminum salt ( $\left.\mathrm{Al}_{2} \mathrm{Cl}_{6}\right)$ into sisal fibers and heat-treat the structure to convert it into alumina fibers. The sisal fibers were pre-treated in $\mathrm{NaOH}$, immersed in a saturated solution of $\left(\mathrm{Al}_{2} \mathrm{Cl}_{6}\right)$ and sintered between $1400{ }^{\circ} \mathrm{C}$ and $1650{ }^{\circ} \mathrm{C}$. The resulting fibers were then characterized by X-ray diffraction and scanning electronic microscopy. The results revealed the transformation only of the surface of the fiber into $\mathrm{\alpha}_{-} \mathrm{Al}_{2} \mathrm{O}_{3}$ which yielded limited resistance to handling. Improved infiltration methods have been studied to allow transformation of the bulk structure of sisal into alumina thus increasing the mechanical strength of the resulting alumina fibers.
\end{abstract}

Keywords: alumina fibers, sisal, biotemplating.

\section{INTRODUÇÃO}

A fibra de sisal é uma das fibras naturais mais utilizadas em escala mundial. No Brasil, ela foi introduzida pelos portugueses, que a importaram da Índia, durante o século XV e cultivada em larga escala a partir do século XX [1]. O 


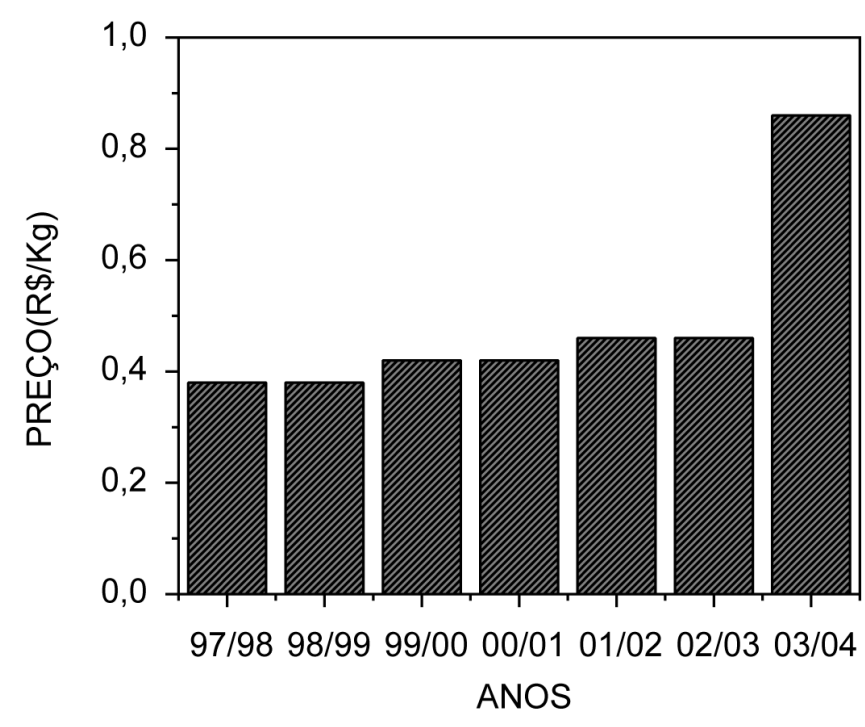

Figura 1: Evolução do preço mínimo da fibra de sisal beneficiada. [Figure 1: Evolution of the minimum market price of washed and brushed sisal fiber.]

plantio do sisal no Brasil concentra-se na região Nordeste, principalmente nos estados da Bahia, Paraíba e Rio Grande do Norte. Neste último, o sisal é cultivado em áreas de pequenos produtores. As condições de clima e solo nessas regiões são pouco favoráveis, resultando em poucas ou nenhuma alternativa para a exploração de outras culturas com resultados econômicos [2]. O sisal é uma cultura resistente e ecológica, para a qual não são utilizados defensivos agrícolas ou adubos químicos. Ela oferece um produto limpo, livre de resíduos químicos, conhecido como sisal orgânico [3]. Atualmente existe uma preocupação dos produtores de sisal do Rio Grande do Norte em relação à revitalização dessa cultura. A formação de uma cooperativa fortaleceu a obtenção de recursos para equipamentos, incentivos e a especialização de mão-de-obra.

As fibras de sisal, como as fibras vegetais em geral, constituem-se, basicamente, de celulose, hemicelulose e lignina, sendo chamadas alternativamente de fibras ligninocelulósicas [04]. Normalmente, o sisal é utilizado na confecção de produtos artesanal e manufaturado incluindo cordas, tapetes e mantas para a indústria automobilística. A industrialização de produtos do sisal tem contribuído para um avanço significativo do preço mínimo da fibra beneficiada (Fig. 1). A necessidade de agregar valores aos produtos manufaturados a partir do sisal contribui não só para o desenvolvimento científico e tecnológico da região, como também para a geração de renda para a população dos municípios potiguares produtores de sisal.

Uma alternativa para a obtenção de produtos com alto valor agregado a partir do sisal é o aproveitamento da estrutura da fibra de sisal para produção de fibras de cerâmicas avançadas incluindo alumina e outros óxidos, carbetos ou nitretos por meio de biomodelagem. Esse método consiste na conversão microestrutural de bioestruturas em materiais cerâmicos com manutenção dos mesmos aspectos morfológicos do material natural, incluindo tubos, poros unidirecionais ou fibras. Essa técnica oferece a possibilidade de se produzir uma ampla variedade de produtos cerâmicos sem a utilização de equipamentos especiais para a produção de diferentes morfologias [5]. A formação de fibras cerâmicas a partir de substratos de fibras naturais tem sido estudada por diversos grupos de pesquisa. Fibras de $\mathrm{SiC}, \mathrm{Si}_{3} \mathrm{~N}_{4}, \mathrm{Al}_{2} \mathrm{O}_{3}$ $\mathrm{SiO}_{2}, \mathrm{Al}_{2} \mathrm{O}_{3}, \mathrm{Al}_{2} \mathrm{O}_{3}-\mathrm{Fe}_{2} \mathrm{O}_{3}$ tem sido produzidas a partir de casca de arroz, fibras de juta, sisal e casca de coco [6]. Particularmente, a obtenção de carbeto de silício partindo de estruturas ligninocelulósicas, através de diferentes rotas de processos, tais como: infiltração de silício no estado líquido em madeira pirolizada, infiltração de gases de $\mathrm{SiO}$ e Si, infiltrações com polímeros e monômeros que contém silício [7]. Atualmente vários grupos pesquisam a obtenção de cerâmicas biomórficas de alumina, mulita e zircônia, obtidas a partir de ratam e pinheiro através do processo de infiltração sol-gel e suspensões de nanopós, onde o processo consiste em infiltrações a vácuo e posterior oxidação em temperaturas elevadas. Em anos recentes diferentes técnicas de biomodelagem estão sendo desenvolvidas para conversão de estruturas bio-orgânicas em cerâmicas biomórficas [8, 9].

\section{MATERIAIS E MÉTODOS}

As fibras de sisal utilizadas neste trabalho foram fornecidas por produtores do estado do Rio Grande do Norte, especificamente da região do Mato Grande. Apesar das fibras serem limpas e escovadas, houve a necessidade de um prétratamento com solução de $\mathrm{NaOH}$ a $0,2 \mathrm{M}$ para remoção de impurezas aderidas à superfície das fibras. Desta forma, procurou-se garantir a desobstrução dos canais de infiltração para posterior tratamento químico com sal de alumínio $\left(\mathrm{AlCl}_{3}\right)$. Após tratamento em meio básico, as fibras foram lavadas com água deionizada e imersas em uma solução saturada de $\mathrm{AlCl}_{3}$ por um período de 24 horas. As fibras de sisal saturadas foram tratadas termicamente em temperaturas que variaram de $1400{ }^{\circ} \mathrm{C}$ a $1650{ }^{\circ} \mathrm{C}$. Com o objetivo de se obter fibras com melhores propriedades mecânicas estão sendo estudadas novas técnicas de infiltração, que neste trabalho foram infiltração sol de alumina e infiltração de nanopós de alumínio. Um sol de alumina consiste de uma dispersão de partículas coloidais em um solvente, originadas da diluição aquosa (água e isopropanol) de um precursor organo-metálico de alumínio que neste trabalho foi utilizado isopropóxido de alumínio $\left(\mathrm{Al}\left(\mathrm{OC}_{3} \mathrm{H}_{7}\right)_{3}\right)$ e o estado da suspensão sol foi determinado por variáveis do processo como: o pH, a temperatura de hidrólise, e concentração dos precursor. A infiltração de nanopós consiste na evaporação de pó de alumínio na estrutura da fibra e posterior oxidação. O material resultante foi caracterizado por microscopia eletrônica de varredura utilizando-se um equipamento Philips XL-30 e por difratometria de raios X $\left(10^{\circ} \leq 2 \theta \leq\right.$ $80^{\circ}$ ) utilizando-se um equipamento Siemens modelo D5000 e radiação $\mathrm{CuK} \alpha$. 

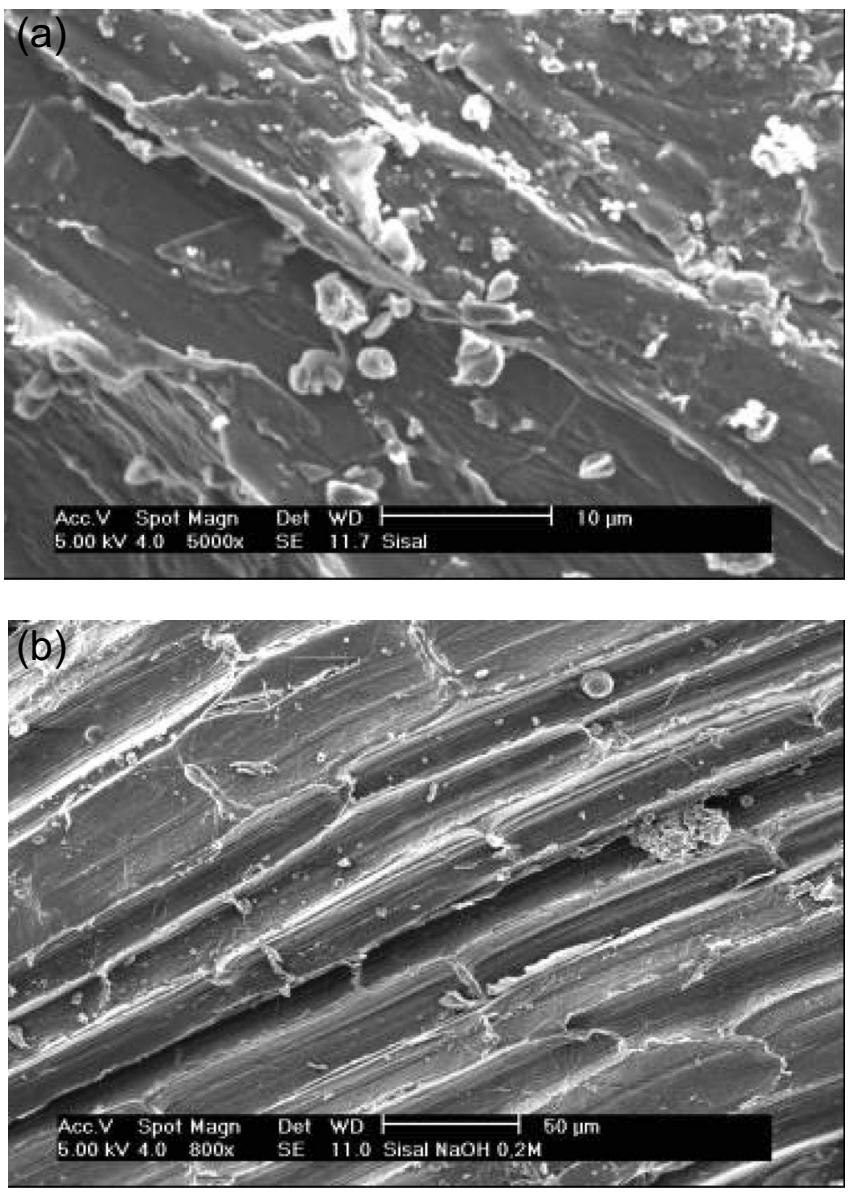

Figura 2: Micrografia da fibra de sisal (a) "in natura" e (b) prétratada com $\mathrm{NaOH}$.

[Figure 2: SEM micrograph of sisal fiber (a) in natura and $(b)$ pretreated in $\mathrm{NaOH}$.]

\section{RESULTADOS E DISCUSSÃO}

A análise superficial da fibra de sisal em seu estado natural (Fig. 2a), revelou a necessidade de pré-tratamento químico, devido a grande quantidade de impurezas, ou seja, partículas que aderem às fibras durante as etapas de extração e beneficiamento das fibras. A limpeza das fibras deu-se por imersão em solução básica, conforme descrito anteriormente, e mostrou-se eficaz na remoção das impurezas e desobstrução dos canais para posterior infiltração salina. Uma típica superfície de fibra de sisal levada para enxerto é mostrada na Fig. 2b.

Fibras saturadas com $\mathrm{AlCl}_{3}$ foram sinterizadas entre $1400{ }^{\circ} \mathrm{C} \mathrm{e}$ $1650{ }^{\circ} \mathrm{C}$. A conversão do sisal em $\alpha-\mathrm{Al}_{2} \mathrm{O}_{3}$ ocorreu já a partir de $1400{ }^{\circ} \mathrm{C}$, conforme mostra o difratograma da Fig. 3. Conforme pode ser exemplificado pela micrografia da Fig. 4, a formação das fibras de alumina reproduziu a superfície da fibra de sisal. As fibras sinterizadas nesta temperatura eram frágeis e não resistiam ao manuseio, o que impede sua utilização na posterior fabricação de mantas ou compósitos. Aumentando a temperatura de sinterização

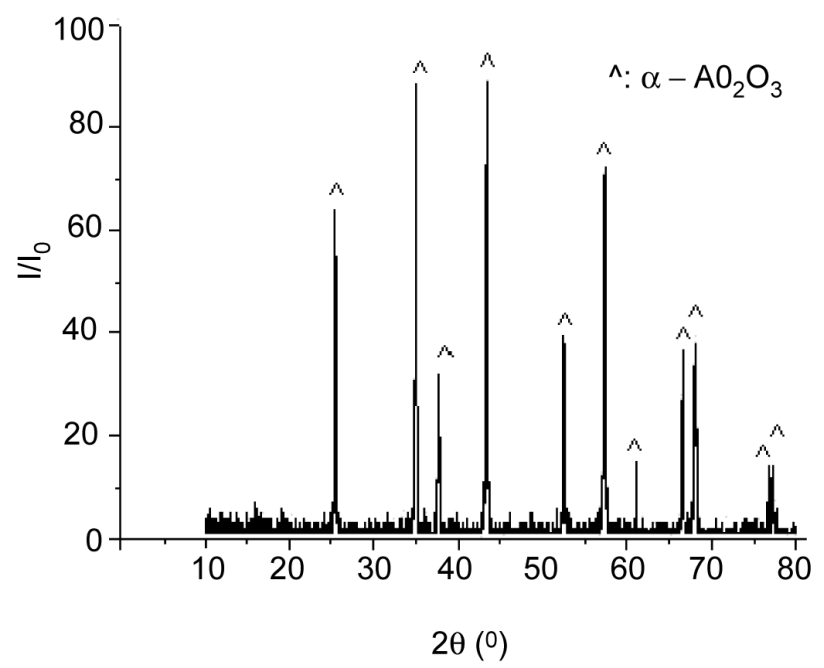

Figura 3: Difratograma de raios $\mathrm{X}$ de fibras de alumina sinterizadas a $1400{ }^{\circ} \mathrm{C}$.

[Figure 3: X-ray diffraction pattern of alumina fibers sintered at $\left.1400{ }^{\circ} \mathrm{C}.\right]$

para $1650{ }^{\circ} \mathrm{C}$, manteve-se a estrutura da $\alpha-\mathrm{Al}_{2} \mathrm{O}_{3}$ com melhor resistência ao manuseio. Mesmo assim, observou-se que as fibras ainda não possuíam resistência mecânica adequada para sua utilização em aplicações de interesse prático. Este fato se deve basicamente à dificuldade em reproduzir-se à estrutura volumétrica da fibra original de sisal em função do tamanho de partículas do sal e da dificuldade de infiltração da solução. Mesmo as fibras sinterizadas a $1650^{\circ} \mathrm{C}$ exibiram somente reprodução superficial da fibra de sisal. Contudo, pôde ser observado neste caso indícios de sinterização na superfície da fibra, como ilustrado pela Fig. 5.

Novos métodos de infiltração têm sido testados em fibras de sisal na busca da reprodução completa da estrutura volumétrica da fibra em sua conversão para alumina. O domínio da metodologia de infiltração aplicada ao sisal

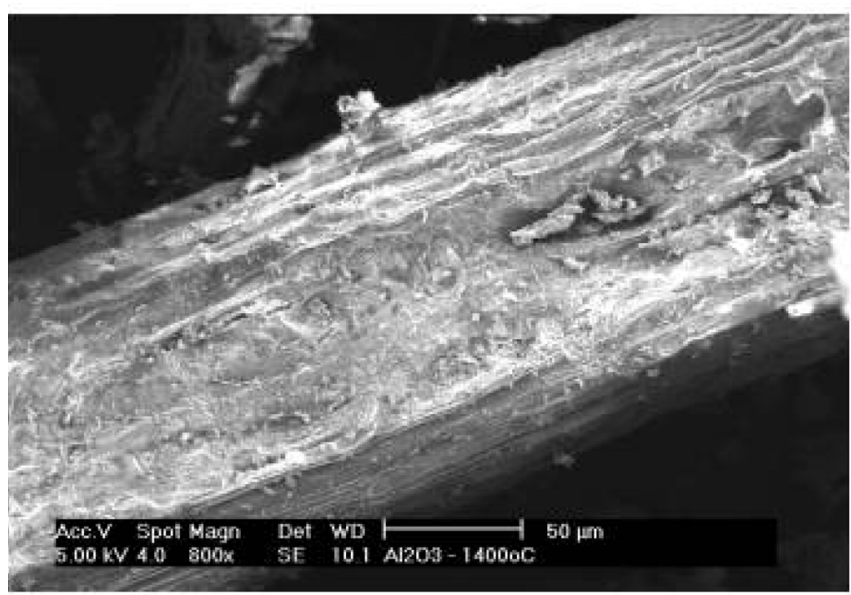

Figura 4: Micrografia eletrônica de fibras de alumina sinterizadas a $1400^{\circ} \mathrm{C}$.

[Figure 4: SEM micrograph of alumina fibers sintered at $1400^{\circ} \mathrm{C}$. ] 


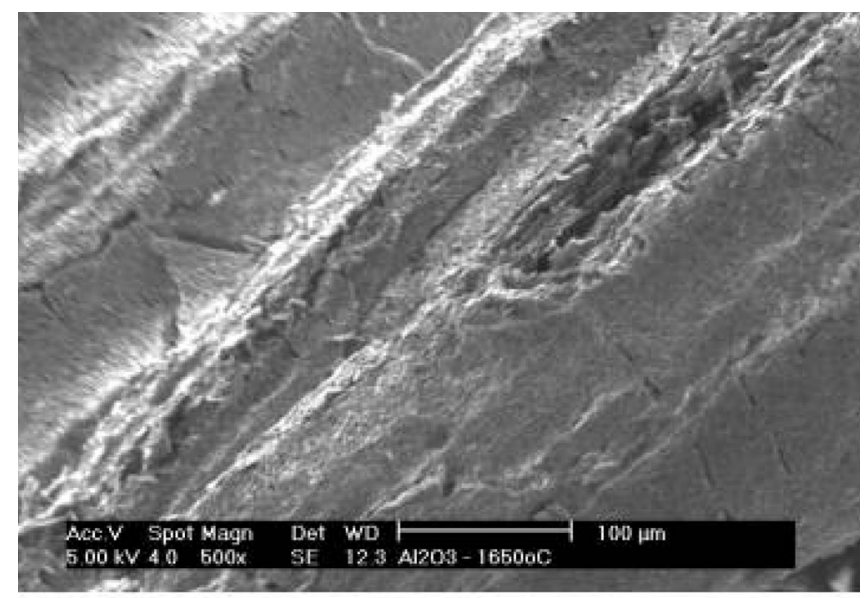

Figura 5: Micrografia eletrônica de fibras de alumina sinterizadas a $1650{ }^{\circ} \mathrm{C}$.

[Figure 5: SEM micrograph of alumina fibers sintered at $1650^{\circ} \mathrm{C}$.]

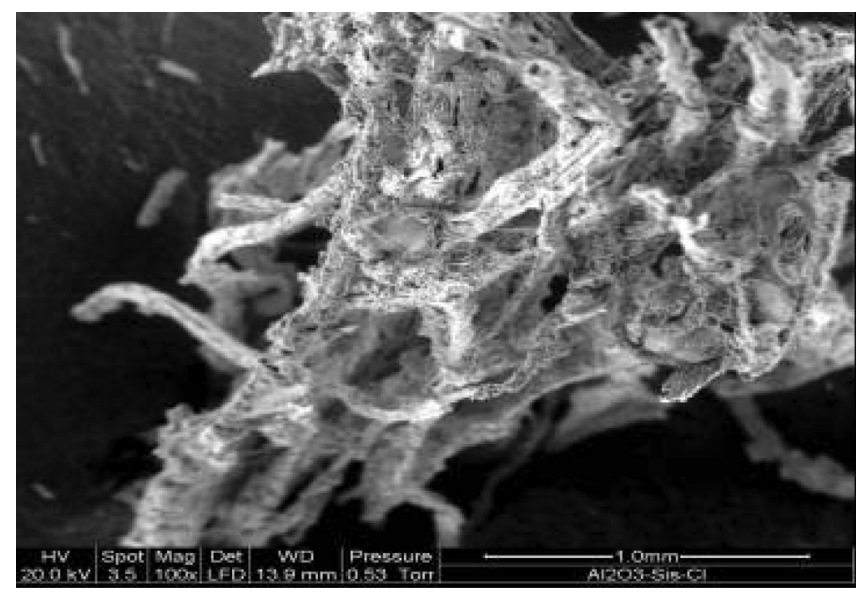

Figura 6: Micrografia eletrônica de fibras de alumina obtidas pelo método sol-gel.

[Figure 6: SEM micrograph of alumina fibers obtained by the solgel method.]

permite a reprodução deste tipo de fibra não só para a produção de fibras e mantas de alumina, mas também de outras cerâmicas óxidas $\left(\mathrm{TiO}_{2}, \mathrm{ZrO}_{2}-\mathrm{Al}_{2} \mathrm{O}_{3}\right.$ e $\mathrm{ZrO}_{2}-\mathrm{Al}_{2} \mathrm{O}_{3}$ $\mathrm{TiO}_{2}$ ) e não-óxidas ( $\mathrm{SiC}$ ). Dentre as técnicas possíveis, estudos estão sendo realizados com infiltrações sol-gel de alumina e de nanopartículas de alumínio.

Para o processo de infiltração sol-gel e de nanopós, as fibras de sisal "in natura" foram secadas em estufa por um período de $24 \mathrm{~h}$ a $130{ }^{\circ} \mathrm{C}$. A solução de alumina foi preparada a partir de isopropóxido de alumínio $\left(\mathrm{Al}\left(\mathrm{OC}_{3} \mathrm{H}_{7}\right)_{3}\right)$ e diluída em água destilada (concentração $0,5 \mathrm{M}$ ). $\mathrm{HNO}_{3}$ foi adicionado para que houvesse a estabilidade da solução e esta não fosse convertida em gel rapidamente, facilitando o processo de infiltração. As fibras de sisal depois de secas foram infiltradas a vácuo com o sol de alumina e posteriormente colocadas para secar a $130{ }^{\circ} \mathrm{C}$ por $2 \mathrm{~h}$. Este processo foi repetido por três vezes. Logo após, as fibras infiltradas foram pirolizadas a $800{ }^{\circ} \mathrm{C}$ por $1 \mathrm{~h}$ em atmosfera de $\mathrm{N}_{2}$ para decomposição dos

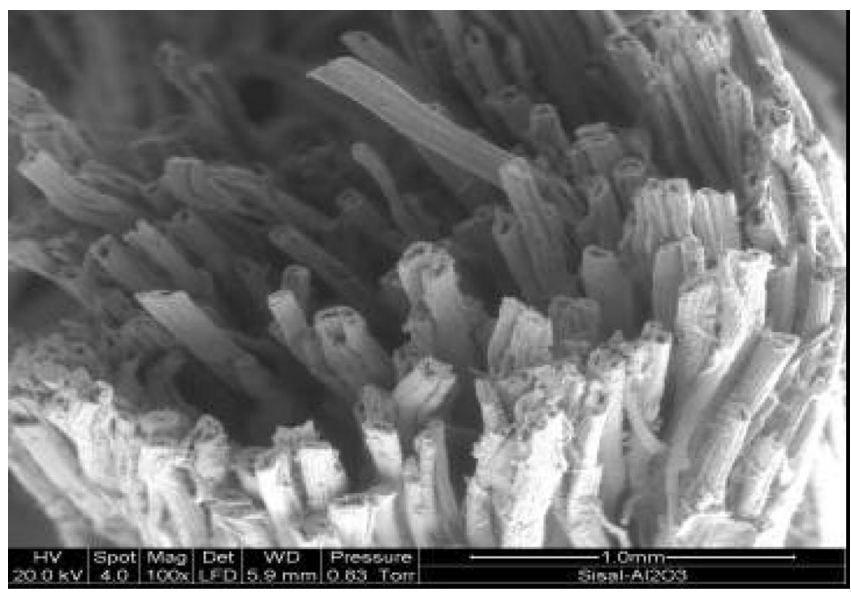

Figura 7: Micrografia eletrônica de fibras de alumina obtidas por infiltração de nanopó de alumínio.

[Figure 7: SEM micrograph of alumina fibers obtained by infiltration of aluminum nanopowder.]

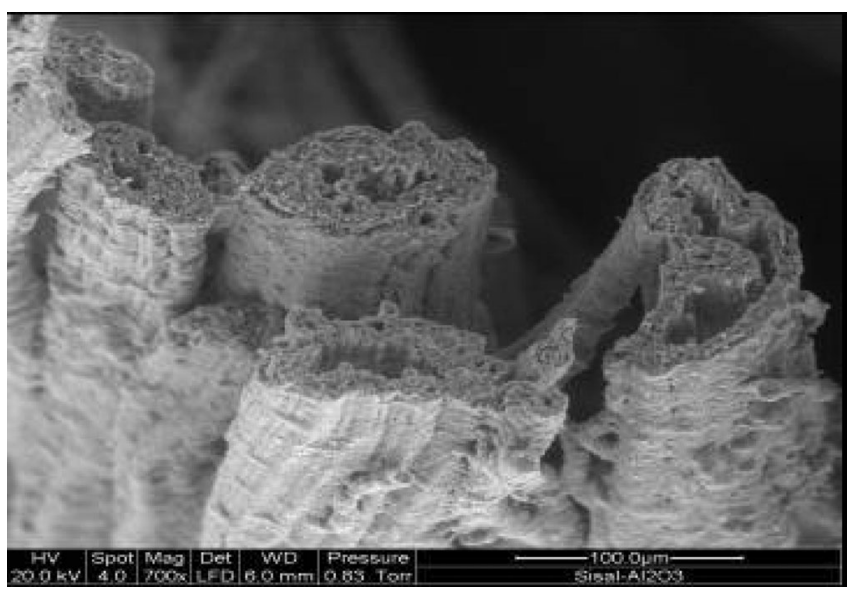

Figura 8: Micrografia da fibra de alumina com reprodução da estrutura interna das fibras originais de sisal.

[Figure 8: SEM micrograph of alumina fibers showing the reproduction of the bulk structure of the original sisal fibers.]

biopolímeros em carbono. Depois de pirolizadas, estas foram infiltradas novamente e oxidadas a $1650{ }^{\circ} \mathrm{C}$. A Fig. 6 mostra as fibras de alumina obtidas pelo processo de infiltração sol de alumina, podendo-se observar que não ocorreu infiltração homogênea na estrutura da fibra e não houve reprodução da fibra de sisal em fibra de alumina, obtendo-se novamente fibras de alumina com baixas resistências mecânicas se comparadas com as fibras obtidas no processo de infiltração de sal de alumínio.

As infiltrações de nanopós de alumínio foram feitas com as fibras sisal pirolizadas. Estas foram convertidas em carbono, mantendo-se a microestrutura da fibra de sisal. As fibras pirolizadas foram infiltradas a partir da evaporação do pó de alumínio na estrutura em atmosfera de argônio a $1600{ }^{\circ} \mathrm{C}$ por $2 \mathrm{~h}$ e posteriormente oxidadas a $1650{ }^{\circ} \mathrm{C}$. Fibras de alumina obtidas a partir do processo de infiltração de nanopós revelaram a reprodução completa da microestrutura, como ilustrado pelas Figs. 7 e 8, melhorando-se, assim, suas 
propriedades mecânicas em comparação com as fibras obtidas pelos métodos descritos anteriormente. Esses resultados comprovaram a eficiência do processo de infiltração de nanopós de alumínio aplicado a sisal, conseguindo-se reproduzir a microestrutura interna das fibras em virtude da penetração homogênea das nanopartículas. A aplicação deste método ao sisal está sendo estudada com maior detalhamento na produção de pré-formas prensadas e mantas.

\section{CONCLUSÕES}

As fibras de sisal foram infiltradas com sal de alumínio, produzindo fibras de alumina após a sinterização entre $1400{ }^{\circ} \mathrm{C} \mathrm{e}$ $1650{ }^{\circ} \mathrm{C}$. Em decorrência da presença de um grande número de pequenos tubos capilares na microestrutura das fibras de sisal, não ocorreu infiltração homogênea da solução salina, resultando em fibras com baixo índice de impurezas, mas com resistência insuficiente ao manuseio. Novos métodos de infiltração, incluindo sol-gel a partir de solução de isopropóxido de alumínio e infiltração de nanopós de alumínio estão sendo investigados. Fibras de alumina têm sido obtidas a partir do sisal pelos dois processos, no entanto, a reprodução adequada da estrutura interna da fibra de sisal foi conseguida apenas pela infiltração de nanopartículas, em virtude de infiltração homogênea, garantindo melhor resistência mecânica das fibras produzidas em relação aos processos anteriores.

\section{AGRADECIMENTOS}

Os autores agradecem a Capes, DAAD e CNPq pelo financiamento deste trabalho de pesquisa.

\section{REFERÊNCIAS}

[1] P. S. Mukherjee, K. G. Satyanarayana, J. Mater. Sci. 19 (1984) 3925-3934.

[2] Secretaria de Agricultura, Irrigação e Reforma Agrária da Bahia, Coordenação de Economia Rural, O Sisal na Bahia, Salvador (1991) 41p.

[3] E. Leal, G. B. Silva, I. C. Costa, L. G. Silva, M. Pereira, O. M. Queiroga, O. V. Miranda, Sistema agroindustrial do sisal na Paraíba, Campina Grande, Universidade Federal da Paraíba (1997) 41p.

[4] D. Fengel, G. Wegener, Wood chemistry, ultrastructure, reactions, Walter de Gruyter, Berlin (1984).

[5] H. Sieber, C. Hoffmann, A. Kaindl, P. Greil, Adv. Eng. Mater. 2, 3 (2000) 105-109.

[6] M. Patel, B. K. Padhi, J. Mater. Sci. Lett. 12 (1993) 1234-1235.

[7] E. Vogli, H. Sieber, P. Greil, J. Eur. Ceram. Soc. 22 (2002) 2663-2668.

[8] C. Rambo, Síntese e caracterização de cerâmicas biomórficas, Tese de Doutorado, IPEN-USP, S. Paulo, SP (2001).

[9] H. Sieber, J. Cao, C. Rambo, P. Greil, Manufacturing of porous oxide ceramics from bioorganic preforms, The $26^{\text {th }}$ Annual Cocoa Beach Conference of the American Ceramic Society (2002).

(Rec. 07/12/2004, Ac. 18/02/2005) 